\title{
Tensile strength of 67P/Churyumov-Gerasimenko nucleus material from overhangs (Corrigendum)
}

\author{
N. Attree ${ }^{1}$, O. Groussin ${ }^{1}$, L. Jorda ${ }^{1}$, D. Nébouy ${ }^{1}$, N. Thomas ${ }^{2}$, Y. Brouet ${ }^{2}$, E. Kührt ${ }^{3}$, F. Preusker $^{3}$, \\ F. Scholten ${ }^{3}$, J. Knollenberg ${ }^{3}$, P. Hartogh ${ }^{4}$, H. Sierks ${ }^{4}$, C. Barbieri ${ }^{5}$, P. Lamy ${ }^{1}$, R. Rodrigo ${ }^{6,7}$, D. Koschny ${ }^{8}$, \\ H. Rickman ${ }^{9,10}$, H. U. Keller ${ }^{3,11}$, M. F. A'Hearn ${ }^{12}$, A.-T. Auger ${ }^{1}$, M. A. Barucci ${ }^{13}$, J.-L. Bertaux ${ }^{14}$, I. Bertini ${ }^{5}$, \\ D. Bodewits ${ }^{12}$, S. Boudreault ${ }^{4}$, G. Cremonese ${ }^{19}$, V. Da Deppo ${ }^{17}$, B. Davidsson ${ }^{7}$, S. Debei ${ }^{16}$, M. De Cecco ${ }^{18}$, \\ J. Deller ${ }^{4}$, M. R. El-Maarry ${ }^{2}$, S. Fornasier ${ }^{13}$, M. Fulle ${ }^{19}$, P. J. Gutiérrez ${ }^{20}$, C. Güttler $^{4}$, S. Hviid ${ }^{3}$, W.-H Ip ${ }^{21}$, \\ G. Kovacs ${ }^{4}$, J. R. Kramm ${ }^{4}$, M. Küppers ${ }^{22}$, L. M. Lara ${ }^{20}$, M. Lazzarin ${ }^{5}$, J. J. Lopez Moreno ${ }^{20}$, \\ S. Lowry ${ }^{23}$, S. Marchi2 ${ }^{24}$, F. Marzari' ${ }^{5}$, S. Mottola ${ }^{4}$, G. Naletto ${ }^{5,17,15}$, N. Oklay ${ }^{3}$, M. Pajola ${ }^{25}$, I. Toth ${ }^{26}$, \\ C. Tubiana ${ }^{4}$, J.-B. Vincent ${ }^{3}$, and X. Shi ${ }^{4}$
}

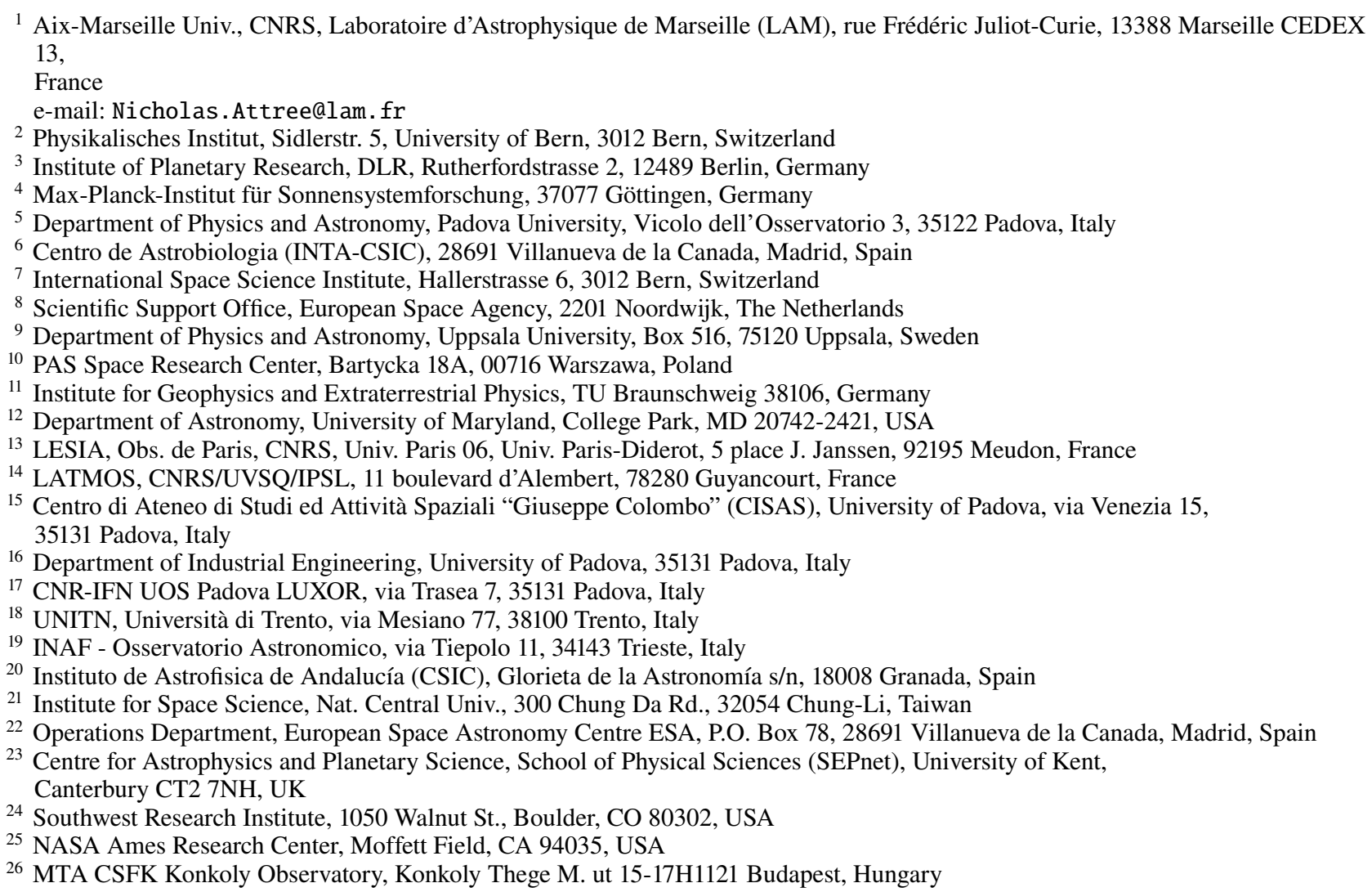

A\&A 611, A33 (2018), https://doi.org/10.1051/0004-6361/201732155

Key words. comets: general - comets: individual: Churyumov-Gerasimenko - methods: observational - errata, addenda

After publication several small errors in the labelled positions of the features described in this paper were found. Correcting these errors results in changes to Table 1 and Figs. 4, 5, and 6. These changes are: the latitude and longitude, as shown in the table and figures, of features 16 and 17 are swapped; and feature 19 was labelled in an incorrect location and has had its latitude, longitude and position in the figures updated. All other data remains the same.

Acknowledgement. We thank Andrew Cooper for spotting the above described errors. 
Table 1. Locations (in the Cheops frame) and properties of each measured overhang.

\begin{tabular}{|c|c|c|c|c|c|c|c|c|}
\hline \multirow{2}{*}{$\frac{\text { No. }}{1}$} & \multirow{2}{*}{$\frac{\text { Latitude }\left(^{\circ}\right)}{16.21}$} & \multirow{2}{*}{$\begin{array}{c}\text { Longitude }\left(^{\circ}\right) \\
-67.47\end{array}$} & \multicolumn{2}{|c|}{$h(\mathrm{~m})$} & \multicolumn{2}{|c|}{$L(\mathrm{~m})$} & \multicolumn{2}{|c|}{$\sigma_{T}(\mathrm{~Pa})$} \\
\hline & & & 109 & +1.8 & 13 & +8.8 & 0.17 & \pm 0.166 \\
\hline 2 & 21.29 & 60.84 & 113 & $\begin{array}{l}-2.4 \\
+6.0 \\
-3.6\end{array}$ & 38 & $\begin{array}{l}-1.2 \\
+8.0 \\
-8.1\end{array}$ & 1.65 & \pm 1.034 \\
\hline 3 & -56.01 & -101.77 & 100 & $\begin{array}{l}-3.6 \\
+11.1\end{array}$ & 15 & $\begin{array}{l}-8.1 \\
+6.7 \\
-68 .\end{array}$ & 0.26 & \pm 0.236 \\
\hline 4 & 40.12 & 23.93 & 109 & $\begin{array}{l}-7.9 \\
+15.2\end{array}$ & 11 & $\begin{array}{l}-0.8 \\
+7.6 \\
-5.1\end{array}$ & 0.15 & \pm 0.154 \\
\hline 5 & 41.94 & 140.53 & 67 & $\begin{array}{l}-13.4 \\
+4.4\end{array}$ & 11 & $\begin{array}{l}-3.1 \\
+4.4\end{array}$ & 0.17 & \pm 0.151 \\
\hline 6 & -56.22 & 58.67 & 106 & $\begin{array}{l}-6.9 \\
+6.2 \\
-99.3\end{array}$ & 6 & $\begin{array}{l}-4.3 \\
+8.1 \\
-5.8\end{array}$ & 0.04 & \pm 0.072 \\
\hline 7 & 32.32 & 161.04 & 41 & $\begin{array}{l}+3.7 \\
-11.6\end{array}$ & 6 & $\begin{array}{l}+3.1 \\
+3.1 \\
-3.1\end{array}$ & 0.08 & \pm 0.071 \\
\hline 8 & -49.00 & 88.69 & 32 & $\begin{array}{l}-1.0 \\
+2.1 \\
-2.8\end{array}$ & 5 & $\begin{array}{l}-3.1 \\
+2.7\end{array}$ & 0.13 & \pm 0.104 \\
\hline 9 & -4.69 & -7.72 & 33 & $\begin{array}{l}-2.8 \\
+22.2 \\
-14.0\end{array}$ & 2 & $\begin{array}{l}-2.3 \\
+2.2 \\
-2.0\end{array}$ & 0.01 & \pm 0.020 \\
\hline 10 & 3.33 & -122.62 & 37 & $\begin{array}{l}-14.0 \\
+1.8 \\
-1.6\end{array}$ & 12 & $\begin{array}{l}-2.0 \\
+2.7 \\
-2.7\end{array}$ & 0.58 & \pm 0.369 \\
\hline 11 & 10.90 & -129.46 & 61 & $\begin{array}{l}-1.0 \\
+2.8 \\
-3.6\end{array}$ & 11 & $\begin{array}{l}-2.1 \\
+4.1 \\
-4.1\end{array}$ & 0.25 & \pm 0.195 \\
\hline 12 & 20.43 & 150.23 & 95 & $\begin{array}{l}-3.0 \\
+8.2 \\
-47.1\end{array}$ & 8 & $\begin{array}{l}-4.1 \\
+4.5 \\
-2.4\end{array}$ & 0.08 & \pm 0.091 \\
\hline 13 & 41.99 & 7.99 & 32 & $\begin{array}{l}-41.1 \\
+1.6\end{array}$ & 7 & $\begin{array}{l}+2.4 \\
+2.7\end{array}$ & 0.18 & \pm 0.138 \\
\hline 14 & -20.69 & 17.02 & 43 & $\begin{array}{l}-6.1 \\
+2.3 \\
-11.4\end{array}$ & 7 & $\begin{array}{l}+3.1 \\
+3.2 \\
-3.2\end{array}$ & 0.13 & \pm 0.114 \\
\hline 15 & 68.89 & -159.29 & 65 & $\begin{array}{l}-11.4 \\
+1.6 \\
-2.7\end{array}$ & 11 & $\begin{array}{l}-3.2 \\
+4.7 \\
-4.8\end{array}$ & 0.25 & \pm 0.209 \\
\hline 16 & -33.69 & 117.56 & 42 & $\begin{array}{l}-2.1 \\
+2.8 \\
-2.8\end{array}$ & 9 & $\begin{array}{l}-4.8 \\
+2.9 \\
-2.8\end{array}$ & 0.28 & \pm 0.204 \\
\hline 17 & -19.06 & 99.47 & 47 & $\begin{array}{l}-2.8 \\
+3.8 \\
-4.5\end{array}$ & 9 & $\begin{array}{l}-2.8 \\
+2.7 \\
-2.8\end{array}$ & 0.19 & \pm 0.150 \\
\hline 18 & 39.06 & -125.97 & 48 & $\begin{array}{l}-4.5 \\
+2.3 \\
-2.7\end{array}$ & 8 & $\begin{array}{l}-2.8 \\
+3.1 \\
-2.8\end{array}$ & 0.22 & \pm 0.170 \\
\hline 19 & -18.12 & 113.00 & 56 & $\begin{array}{l}-2.1 \\
+2.8 \\
-5.8\end{array}$ & 10 & $\begin{array}{l}-2.8 \\
+3.1 \\
-3.1\end{array}$ & 0.23 & \pm 0.186 \\
\hline 20 & -21.81 & -28.84 & 9 & $\begin{array}{l}-1.0 \\
-1.6\end{array}$ & 6 & $\begin{array}{l}+1.7 \\
-1.7\end{array}$ & 0.77 & \pm 0.418 \\
\hline
\end{tabular}

Notes. $h$ and $L$ are the heights and depths, as directly measured from the profiles, while $\sigma_{T}$ is derived by numerically integrating the profile shape.

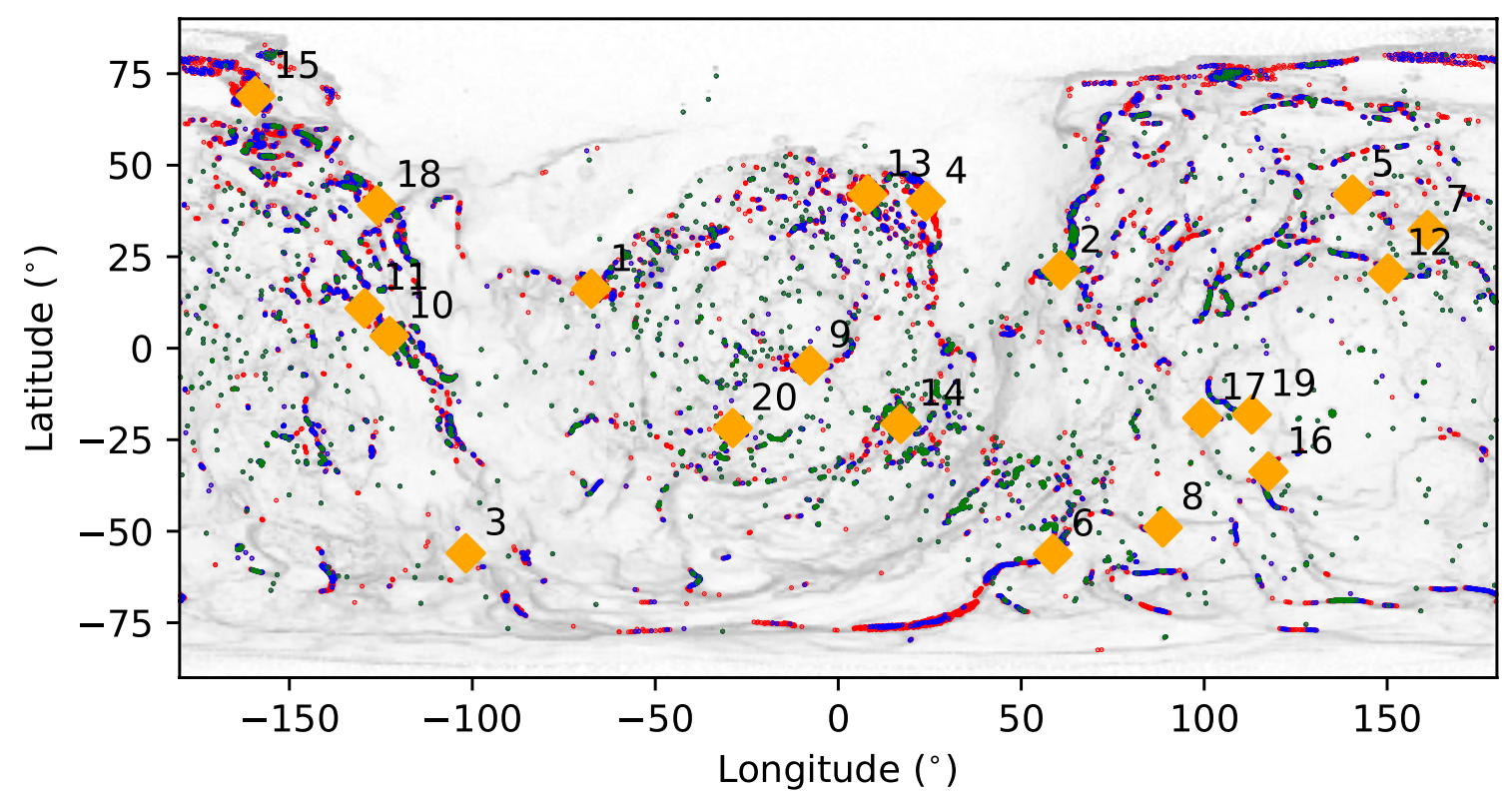

Fig. 4. Location of the measured overhangs (orange diamonds). Also shown are high-slope facets on the shape model with the colour scheme: green $\geq 100^{\circ}, 100^{\circ}>$ blue $\geq 90^{\circ}$, and $90^{\circ}>$ red $\geq 85^{\circ}$. 


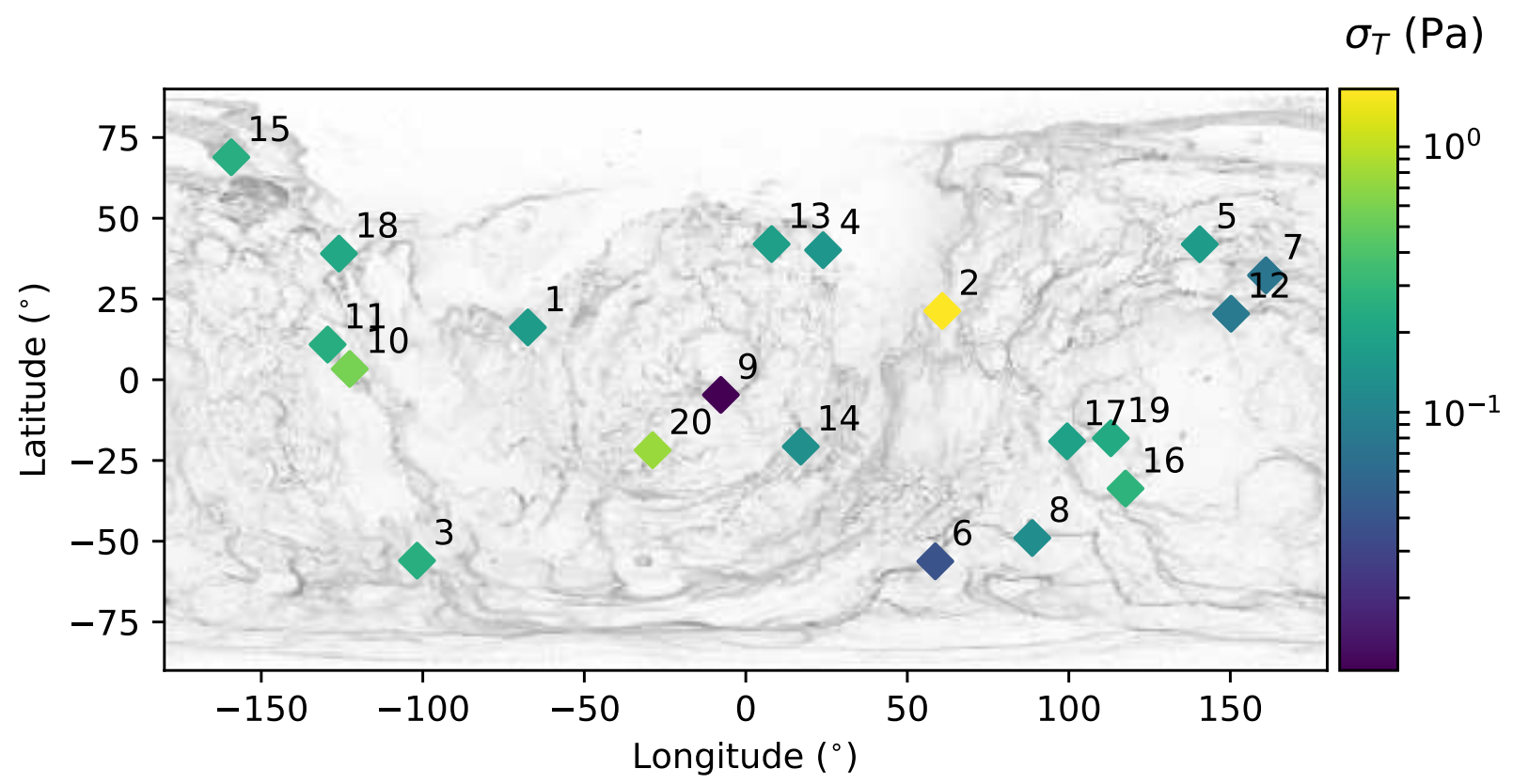

Fig. 5. Location of the measured overhangs. The colour scheme shows the tensile strength (unscaled) on a log scale.
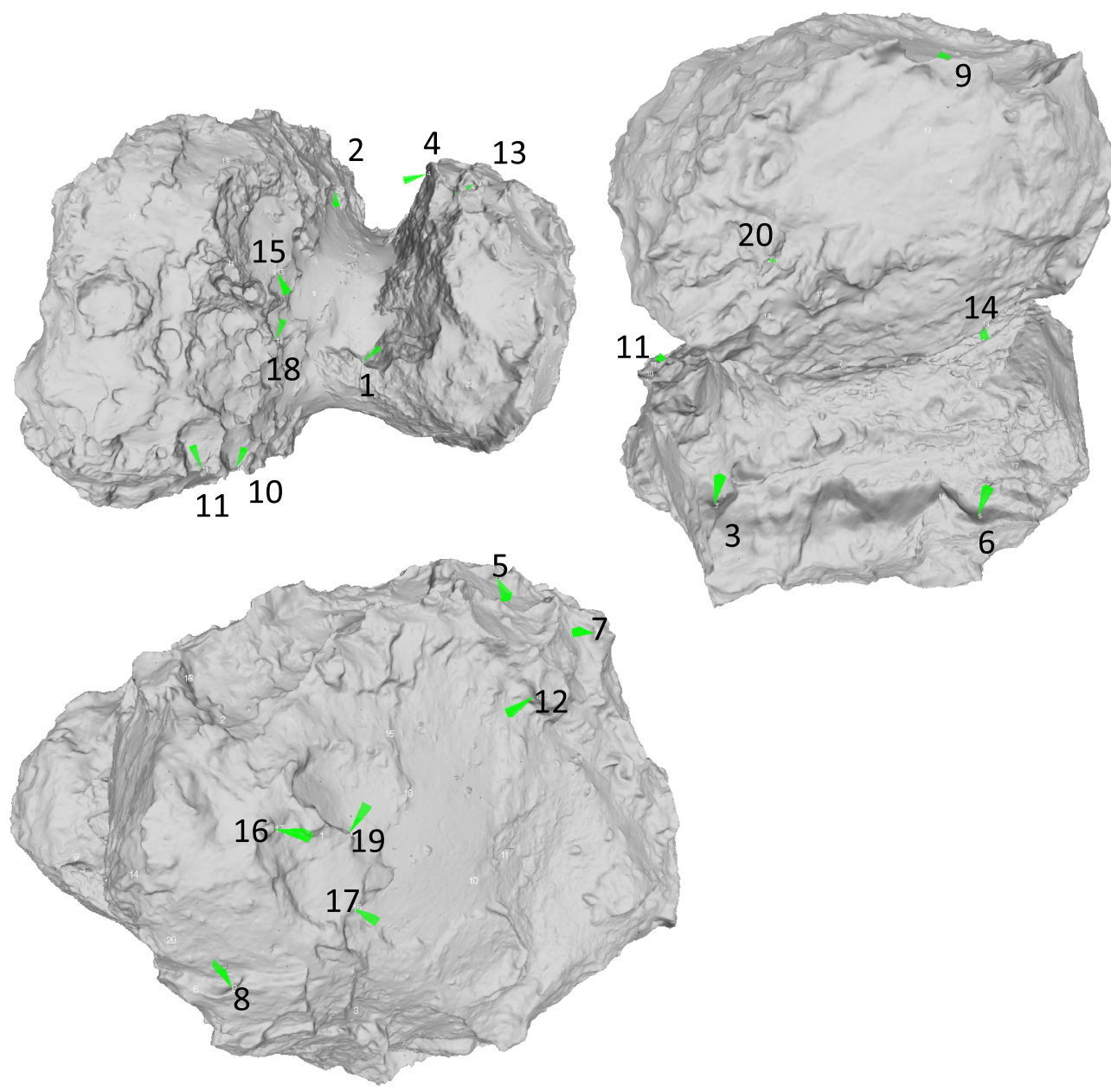

Fig. 6. Location of the measured overhangs on the shape model. 